\title{
Experimentally and Numerically Analyzed Piezoelectric Characteristics of PZT Ceramics
}

\author{
Lale Yang $^{1}$, Hirokazu Nagano ${ }^{2} \&$ Mitsuhiro Okayasu $^{1}$ \\ ${ }^{1}$ Graduate School of Natural Science and Technology, Okayama University, 3-1-1 Tsushimanaka, Kita-ku, \\ Okayama, 700-8530, Japan \\ ${ }^{2}$ Disaster Prevention and City Development Section, Arakawa City Office, 2-2-3 Arakawa, Arakawa-ku, Tokyo, \\ 116-8501, Japan \\ Correspondence: Mitsuhiro Okayasu, Graduate School of Natural Science and Technology, Okayama University, \\ 3-1-1 Tsushimanaka, Kita-ku, Okayama, 700-8530, Japan. E-mail: okayasu@okayama-u.ac.jp
}

Received: August 31, 2018

Accepted: October 18, 2018

Online Published: December 12, 2018

doi: $10.5539 /$ jmsr.v8n1p10

URL: https://doi.org/10.5539/jmsr.v8n1p10

\begin{abstract}
The electric generation characteristics of the PZT piezoelectric ceramics were examined experimentally and numerically. In this case, cyclic bending loading was applied to the PZT ceramics: bulk and thin-film membrane. Electric voltage increased with increasing the strain value. There was a sharp initial increase in positive voltage for the PZT ceramics as bending load was applied. Following this initial increase stage, a rapid fall to zero occurred. The reduction rate of the positive voltage was changed depending on the rigidly of the PZT ceramic: the higher the reduction rate, the higher the rigid of the ceramic. The electrical voltage from the circular membrane PZT ceramic during cyclic loading showed the higher level compared to that for the bulk one, in which the flexibility of the PZT ceramic was significant factor to obtain the high electric voltage. The variation of the electric voltages was estimated numerically, which was relatively in good agreement with the experimental one.
\end{abstract}

Keywords: piezoelectric ceramic, lead zirconate titanate ceramic, experiment, numerical analysis

\section{Introduction}

The energy conversion of electrical and mechanical sources is an important aspect in our current life for power generators. In particular, piezoelectric materials are prospective materials for energy conversion, since they have a good electrical-mechanical coupling effect (Lu, Lee, \& Lim, 2003). In recent years, electrical power generation from a variety of sources has received a lot of attention as a result of environmental and energy issues. One of the representative energy harvesting systems is that made by vibrating structures, which transform mechanical energy into electrical energy. There are several commercial piezoelectric materials, such as lead, bismuth, barium and lead zirconate tinatate (PZT) ceramic; and especially the PZT ceramic has been widely employed because of the better piezoelectric properties.

Today, harvested energy systems have been developed by several engineers as new energy systems in which PZT ceramic plates are embedded under the floor or ground, e.g., roads, bridges, houses, and wicket gates. In this instance, the energy in the pavement caused by vehicles and gravity can be harvested using piezoelectric transducers (Zhao, Yu, \& Ling, 2010); moreover, a related energy could be obtained via the walking motion of the human body, e.g., a heel-strike generator (Howells, 2009). It is considered that energy harvesting systems are expected to be part of the energy sources in our society. However, there would be technical problems in this system, caused by the low efficiency of the electric energy generation: the mean power from the system is much less than its target of $0.5 \mathrm{~W}$. To solve this, several researchers have attempted to investigate the influence of the vibration condition on the electrical generation characteristics, in which loading conditions were altered, such as frequency and loading wave mode. Shu and Lien have investigated the harvested power generation characteristics under different vibrating conditions (frequency and acceleration), mechanical damping ratio, and electromechanical coupling coefficient. Theoretical predictions have also been made and have been found to be in good agreement with the experimental observations (Shu \& Lien, 2006). PZT ceramics have been used for associated experiments, but many PZT ceramics cannot be employed due to their low machinability and their high price. Because of these issues, it might be expected that numerical analyses are undertaken instead of an 
experimental approach. Until now, several investigators have used finite element analysis (FEA), in which the efficiency and coupling effects with the pavement of cymbal piezoelectric transducers with various sizes are discussed. The displacement difference at the pavement surface with and without a cymbal is developed in order to consider the coupling effect. The results show that the potential electric energy harvested from the pavement increases with the increment of the diameter of the cymbal (Zhao, Yu, \& Ling, 2010). Liew et al. have proposed an efficient finite element model, which is presented for static and dynamic piezo-thermoelastic analysis under temperature gradient environments using integrated piezoelectric sensor/actuator layers (Liew, He, $\mathrm{Ng}, \&$ Kitipornchai, 2003). A further approach has been performed by Gönandt and Gabbert, who have extended commercial finite element software by including a pool of piezoelectric finite elements to provide solutions in 1D, 2D and 3D (Görnandt \& Gabbert, 2002). Even though several investigators have analyzed the piezoelectric properties of the associated ceramics, there is a lack of the related experimental and numerical approaches. In particular, no clear analysis is obvious for investigation of the piezoelectric properties (electric generation) and for the confirmation of the experimental results under different mechanical loading conditions. Thus, in the present work, an attempt was made to perform the experimental and numerical analysis to understand the electrical generation characteristics of the commercial PZT ceramics.

\section{Experimental Procedures}

\subsection{Sample Preparation}

In the present work, commercial bulk and thin-film piezoelectric membrane PZT $\left(\mathrm{PbZrTiO}_{3}\right)$ ceramics were used. Figure 1 displays the schematic illustration of the PZT ceramics. The circular membrane PZT ceramic is formed with a thin round plate of $\phi 8.0 \times 0.17 \mathrm{~mm}$, attached to a thin brass plate $(\phi 12.2 \times 0.15 \mathrm{~mm})$. The electrode was made by silver-plating on the surface of the PZT ceramic, which created by a firing process in atmosphere before a poling process at high electric voltage. The bulk PZT ceramic is formed with a rectangular rod with the following dimensions: $3 \times 3 \times 40 \mathrm{~mm}$. Like the membrane PZT, the silver based metal was coated on the two opposed surfaces of the bulk PZT ceramic. The material properties of the PZT ceramic and the brass plate are as follows: elastic constant $(L) 82 \mathrm{GPa}$ and Poisson's ratio $(v) 0.3$ for the PZT ceramic; and $L=103 \mathrm{GPa}$ and $v=$ 0.35 for the brass plate. The density of PZT ceramics is approximately $7.68 \mathrm{~g} / \mathrm{cm}^{3}$ and Curie point is $568 \mathrm{~K}$. The piezoelectric coefficient is about $460 \mathrm{pm} / \mathrm{V}$. The main piezoelectric properties (piezoelectric constant $(d)$ and dielectric constant $(\varepsilon)$ ) are indicated in Table 1 .

Table 1. Piezoelectric properties of the PZT ceramics: (a) piezoelectric constant (d) and (b) dielectric constant ( $\varepsilon$ )

(a) Piezoelectric constant

\begin{tabular}{llll}
\hline$d_{31}$ & $d_{33}$ & $d_{15}$ & Others \\
\hline-130 & 260 & 550 & 0 \\
\hline
\end{tabular}

(b) Dielectric constant

\begin{tabular}{lll}
\hline$\varepsilon_{11}$ & $\varepsilon_{33}$ & Others \\
\hline 1600 & 1300 & 0 \\
\hline
\end{tabular}

(a) Circular membrane PZT ceramic
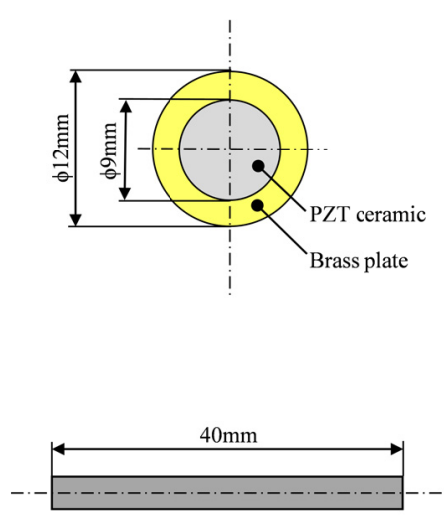

(b) Bulk PZT ceramic

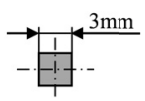

Figure 1. Schematic illustration of the test specimen of the (a) circular membrane and (b) bulk PZT ceramics 


\subsection{Experimental Approach}

To investigate the electric generation characteristics of the PZT ceramics, the cyclic bending load was mechanically applied to the PZT ceramic at different strain levels using an originally designed loading device. Figure 2 indicates a schematic diagram of the test apparatus, consisting of the PZT ceramics, the specimen fixture and the loading machine. In this approach, the PZT ceramic was put on the stage of the fixture, where the cyclic loading was applied at $1 \mathrm{~Hz}$ for about 100 cycles under the strain control, where a square wave mode was selected. Note that the maximum strain value was determined on the basis of their fracture strains. This test was carried out using a $50 \mathrm{kN}$ screw driven type universal testing machine. The electric voltage was continuously monitored for 10 cycles using a digital multimeter (8846A, Fluke).
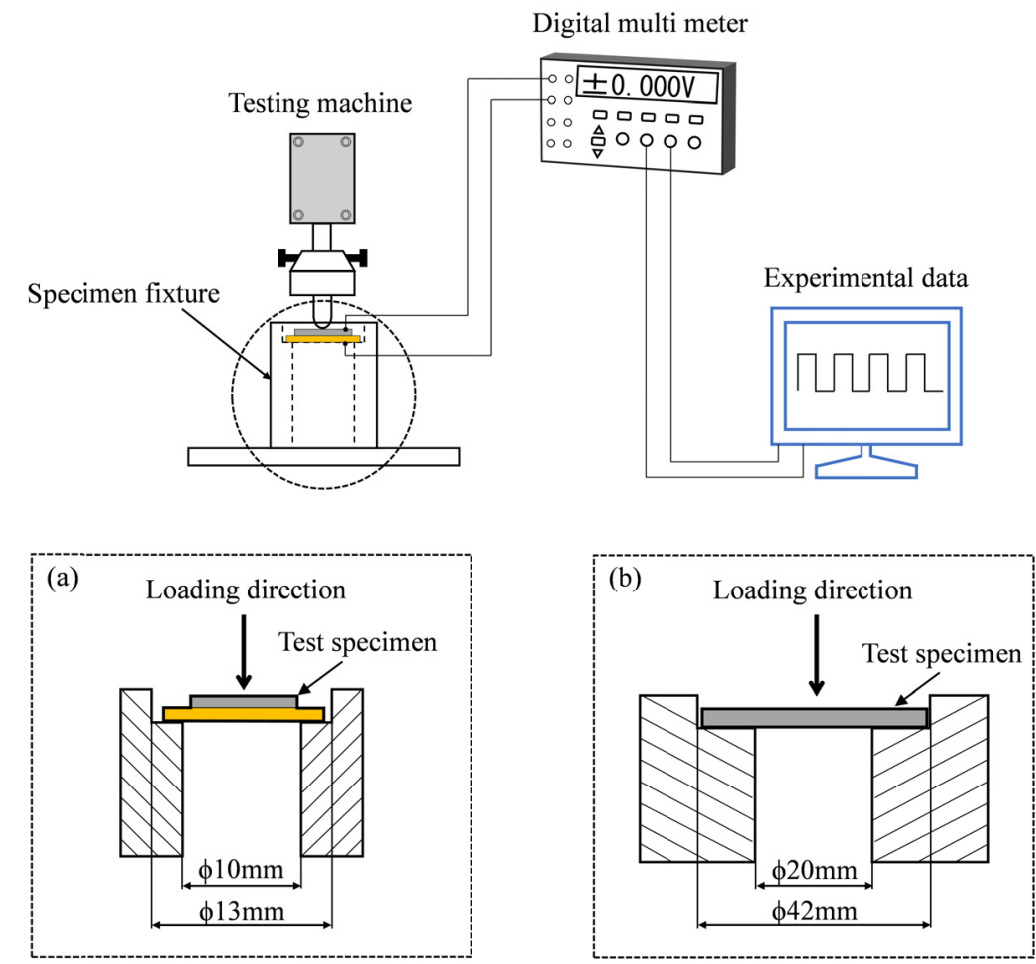

Figure 2. Schematic diagram of the test apparatus, consisting of the test specimen (a) circular membrane PZT ceramics (b) bulk PZT ceramics and the testing machine

\subsection{Numerical Approach}

Numerical analysis was executed using commercial software (MARC 2008) to calculate the electric voltage of the PZT ceramics under the bending loading. Figure 3 shows the model of the test specimens, which designed based upon the specimen geometry shown in Figure 1. Furthermore, the distributions of the electric field intensity under the bending loading are displayed. Two-dimensional analysis with 8-noded quad elements was conducted. The mesh size of both models was determined to be less than $0.01 \mathrm{~mm}$. In this analysis, the bending load was applied to the PZT ceramics based upon the experimental conditions, as described above.

The value of electric displacement $(D)$, i.e., electric energy, can be calculated with the following equation:

$$
D=T d+\varepsilon^{\mathrm{T}} E
$$

where $d$ is the piezoelectric constant, $T$ is the applied stress, $\varepsilon^{T}$ is the permittivity and $E$ is the electric field. If the value of the electric field is much low as high stress is applied, the parameter of $\varepsilon^{\mathrm{T}} E$ could be omitted. In this case, the $D$ value is hence approximated by

$$
D=T d
$$




$$
\left[\begin{array}{l}
D_{1} \\
D_{2} \\
D_{3}
\end{array}\right]=\left[\begin{array}{llllll}
d_{11} & d_{12} & d_{13} & d_{14} & d_{15} & d_{16} \\
d_{21} & d_{22} & d_{23} & d_{24} & d_{25} & d_{26} \\
d_{31} & d_{32} & d_{33} & d_{34} & d_{35} & d_{36}
\end{array}\right]\left[\begin{array}{l}
T_{1} \\
T_{2} \\
T_{3} \\
T_{4} \\
T_{5} \\
T_{6}
\end{array}\right]+\left[\begin{array}{lll}
\varepsilon_{11} & \varepsilon_{12} & \varepsilon_{13} \\
\varepsilon_{12} & \varepsilon_{22} & \varepsilon_{23} \\
\varepsilon_{13} & \varepsilon_{23} & \varepsilon_{33}
\end{array}\right]\left[\begin{array}{l}
E_{1} \\
E_{2} \\
E_{3}
\end{array}\right]
$$

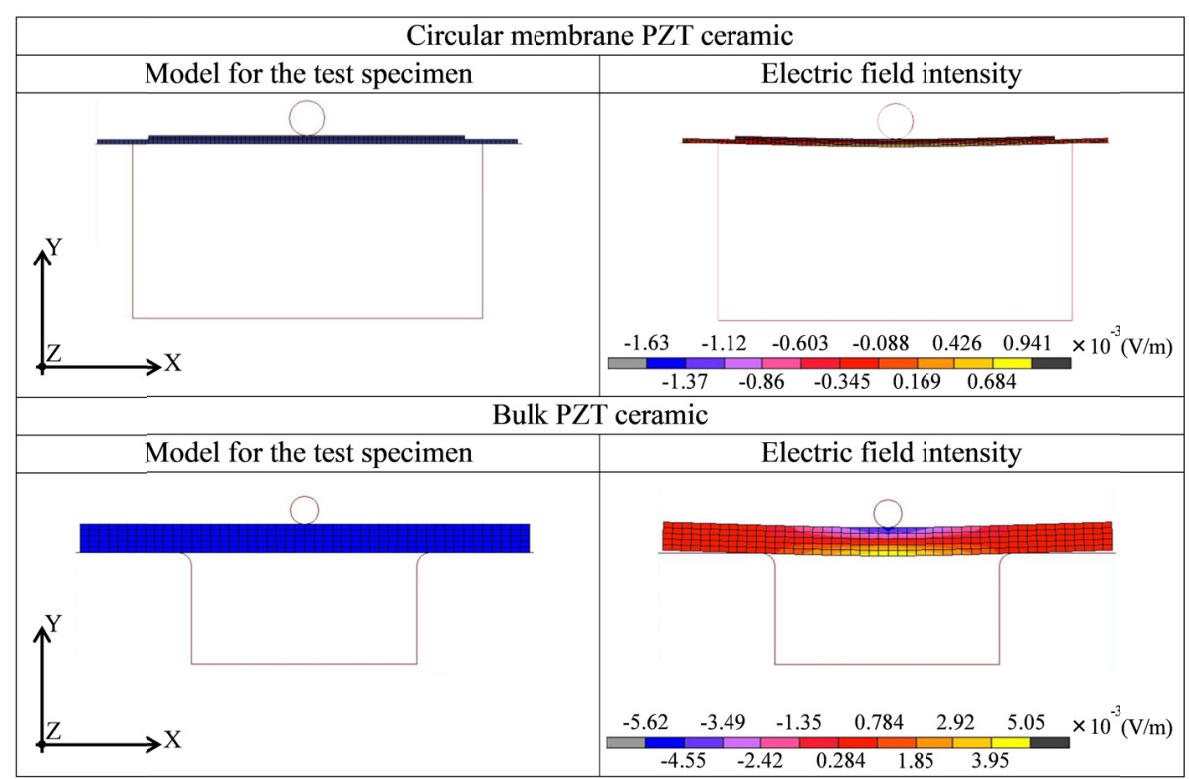

Figure 3 FEA models and the distribution of the electric field intensity of the PZT ceramics

On the other hand, the electric charge $(q)$ of the PZT ceramic can be described as:

$$
q=A D
$$

where $A$ is the area of the PZT ceramic. By combining the above eq. (2) and (3), the electric charge can be expressed by

$$
\begin{gathered}
q \approx A T d(\approx F d) \\
q=\left[A_{1} A_{2} A_{3}\right]\left[\begin{array}{llllll}
d_{11} & d_{12} & d_{13} & d_{14} & d_{15} & d_{16} \\
d_{21} & d_{22} & d_{23} & d_{24} & d_{25} & d_{26} \\
d_{31} & d_{32} & d_{33} & d_{34} & d_{35} & d_{36}
\end{array}\right]\left[\begin{array}{c}
T_{1} \\
T_{2} \\
T_{3} \\
T_{4} \\
T_{5} \\
T_{6}
\end{array}\right]
\end{gathered}
$$

where $F$ is the applied force. The electric charge is related to the electric voltage $(V)$ and braking capacity $\left(C_{d}\right)$ (Fuji Ceramics Co., n.d.):

$$
V=q / C_{d}
$$

The $C_{d}$ value is related with the dielectric constant $(\varepsilon)$ and the shape of the PZT ceramic:

$$
C_{d}=A \varepsilon / l
$$

$$
\left[\begin{array}{lll}
C_{1} & C_{2} & C_{3}
\end{array}\right]=\left[\begin{array}{lll}
A_{1} & A_{2} & A_{3}
\end{array}\right]\left[\begin{array}{lll}
\varepsilon_{11} & \varepsilon_{12} & \varepsilon_{13} \\
\varepsilon_{12} & \varepsilon_{22} & \varepsilon_{23} \\
\varepsilon_{13} & \varepsilon_{23} & \varepsilon_{33}
\end{array}\right] / l
$$

where $l$ is the specimen length. Substituting eqs.(6) and (4) into eq.(5) leads to an expression for the electric voltage as follows:

$$
V=d T l / \varepsilon
$$




$$
\left[\begin{array}{l}
\mathrm{V}_{1} \\
\mathrm{~V}_{2} \\
\mathrm{~V}_{3}
\end{array}\right]=\left[\begin{array}{llllll}
d_{11} & d_{12} & d_{13} & d_{14} & d_{15} & d_{16} \\
d_{21} & d_{22} & d_{23} & d_{24} & d_{25} & d_{26} \\
d_{31} & d_{32} & d_{33} & d_{34} & d_{35} & d_{36}
\end{array}\right]\left[\begin{array}{l}
\mathrm{T}_{1} \\
\mathrm{~T}_{2} \\
\mathrm{~T}_{3} \\
\mathrm{~T}_{4} \\
\mathrm{~T}_{5} \\
\mathrm{~T}_{6}
\end{array}\right] l\left[\begin{array}{llll}
\varepsilon_{11} & \varepsilon_{12} & \varepsilon_{13} \\
\varepsilon_{12} & \varepsilon_{22} & \varepsilon_{23} \\
\varepsilon_{13} & \varepsilon_{23} & \varepsilon_{33}
\end{array}\right]
$$

\section{Results and Discussion}

\section{Experimental approach}

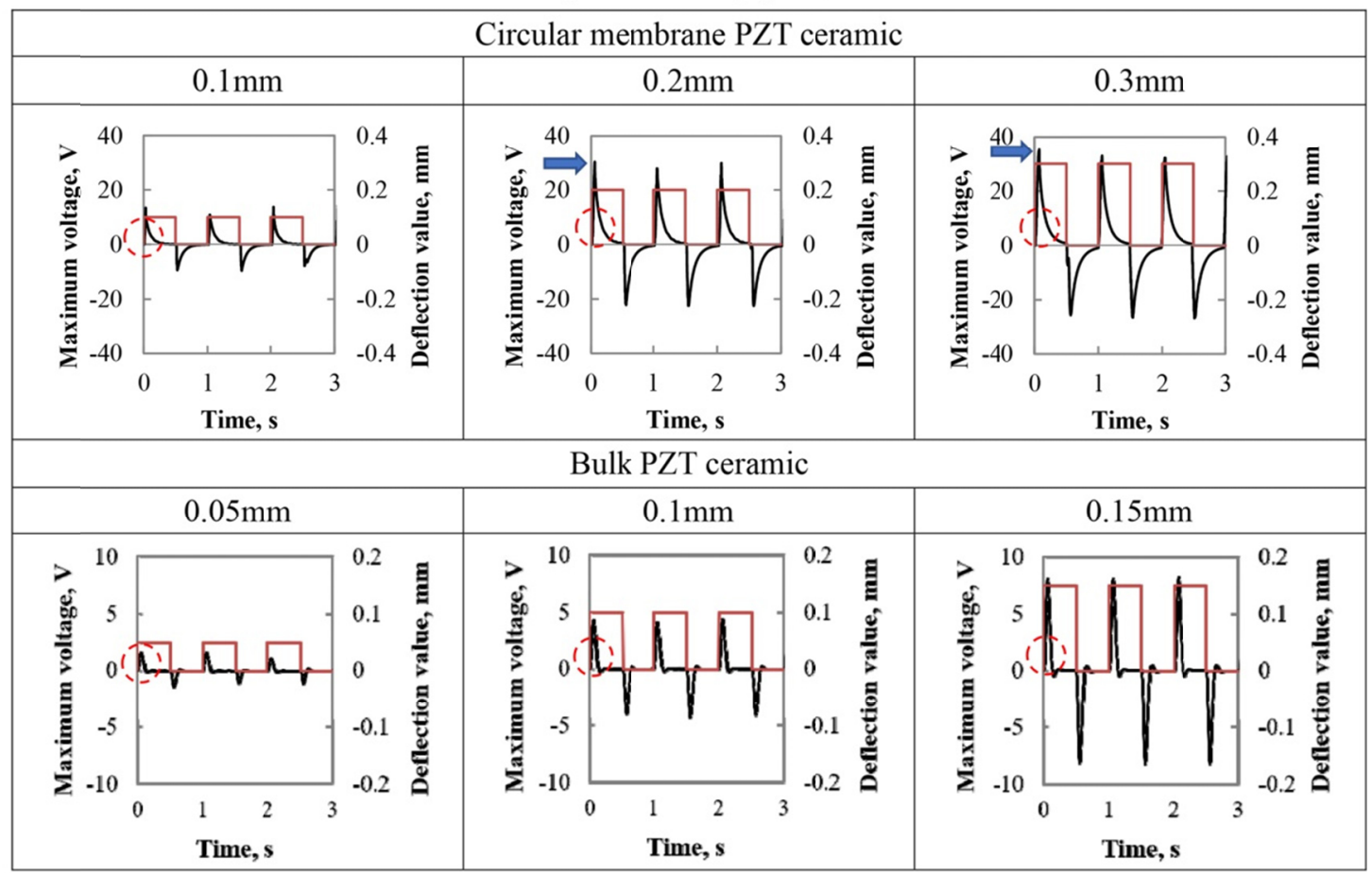

Figure 4 Variations of experimental electric voltages with time for both bulk and circular membrane

Figure 4 displays the variations of the maximum electric voltages with time for both bulk and circular membrane obtained experimentally. It is obvious that, the electric voltage rises sharply to the maximum level as applied load is conducted to the PZT ceramics. In this case, electrons in the PZT ceramic flow from the ceramic, as bending load is applied, resulting in the generation of the electric voltage. The electric voltage decreases to $0 \mathrm{~V}$ even if the bending load is continuously applied, where the electrons could have all flown (Okayasu \& Watanabe, 2015). Interestingly, the reduction rate of electric voltage is different depending on the sample as enclosed by the dashed circles in Figure 4: that decreases rapidly and slowly for bulk and circular membrane PZT ceramic, respectively. In this case, the different reduction rate may be caused by the difference in rigidly of the sample, with regard to, for example, the higher rigid for bulk PZT ceramic makes the higher reduction rate. With the removal of the bending load, a reversal of the electrons to the PZT ceramic occurs, leading to the generation of the negative electric voltage, where the electrons flow into an opposite direction.

It is also seen in Figure 4 that the maximum voltage increases with increasing bending strain value (Okayasu \& Watanabe, 2016), where the high mechanical energy makes high electrical energy. However, the maximum electric voltage is similar level for circular membrane PZT as loaded to $0.2 \mathrm{~mm}$ (Ave. 31V) and $0.3 \mathrm{~mm}$ (Ave. $35 \mathrm{~V}$ ), as indicated by the arrows. This could be affected by the same electron levels that all electrons flow for its electric generation. On the other hand, the electric voltage value for the circular membrane is apparently high compared to that for bulk sample. As seen, the electric voltage for the membrane sample is more than 10 times higher than that for bulk one, which would be attributed to the high flexibility for the membrane sample. 
Figure 5 displays the variation of the electric voltages numerically analyzed for both PZT ceramics. It is clear that the electric voltage increases sharply as the bending load is applied, where the maximum voltage increases with increasing the strain value. The electric voltage drops suddenly after the maximum voltage is obtained, and that value shows the negative value when the applied load is removed. The variation of the electric voltage is relatively similar to that for their experimental ones. This analysis would be applicable for estimation of the electric voltage.

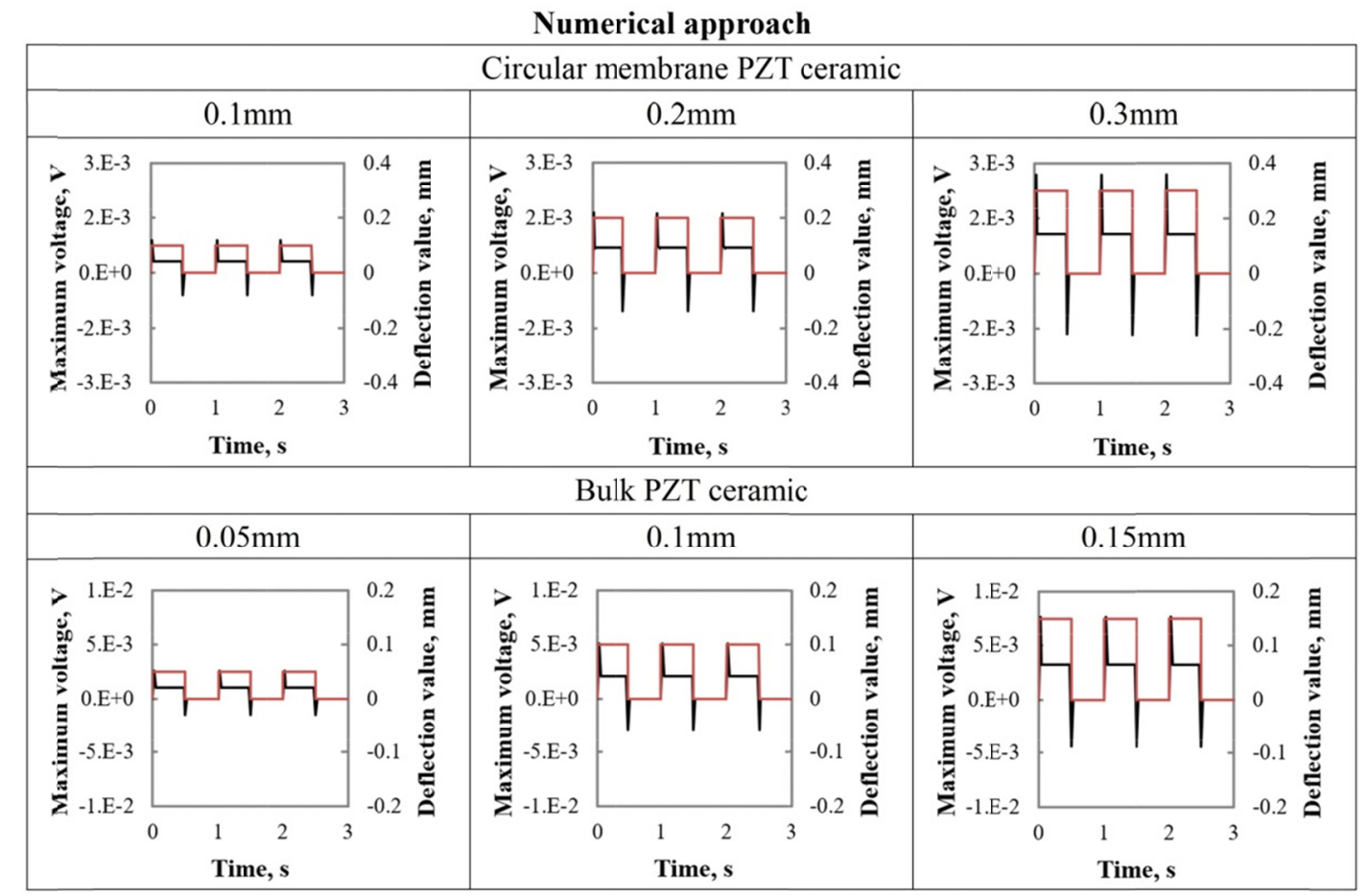

Figure 5 Variations of numerical electric voltages with time for both bulk and circular membrane

\section{Conclusions}

The electrical power generation properties of the PZT piezoelectric ceramics have been examined experimentally and numerically using two different samples, bulk and thin-film membrane. The obtained results are given as follows:

(1) Electric voltage increases with increasing bending strain value. There is a sharp initial increase in positive voltage for both samples as the bending load is applied. Following this initial increase stage, a rapid fall to zero occurs, leading to the generation of increasingly negative voltage when the applied load is removed to zero strain. The reduction rate of the electric voltage is changed depending on the rigidly of the PZT ceramic: the higher the reduction rate, the higher the rigid of the ceramic.

(2) The electric voltage, generated from the circular membrane PZT ceramic during cyclic loading, shows the higher level compared to that for the bulk one. The flexibility of the ceramic sample is significant factor to obtain the high electric voltage. The voltages generated are estimated relatively well by a numerical analysis, which shows the similar variation of the electric voltage obtained experimentally.

\section{References}

Fuji Ceramics Co. (n.d.). Technical Handbook (pp. 12-16).

Görnandt, A., \& Gabbert, U. (2002). Finite element analysis of thermopiezoelectric smart structures. Acta Mechanica, 154(1-4), 129-140.

Howells, C. A. (2009). Piezoelectric energy harvesting. Energy Conversion and Management, 50(7), 1847-1850.

Liew, K. M., He, X. Q., Ng, T. Y., \& Kitipornchai, S. (2003). Finite element piezothermoelasticity analysis and the active control of FGM plates with integrated piezoelectric sensors and actuators. Computational Mechanics, 31(3-4), 350-358. 
Lu, F., Lee, H. P., \& Lim, S. P. (2003). Modeling and analysis of micro piezoelectric power generators for micro-electromechanical-systems applications. Smart Materials and Structures, 13(1), 57-63.

Okayasu, M., \& Watanabe, K. (2015). The electric power generation characteristics of a lead zirconate titanate piezoelectric ceramic under various cyclic loading conditions. Ceramics International, 41(10), $15097-15102$.

Okayasu, M., \& Watanabe, K. (2016). A study of the electric power generation properties of a lead zirconate titanate piezoelectric ceramic. Ceramics International, 42(12), 14049-14060.

Shu, Y. C., \& Lien, I. C. (2006). Analysis of power output for piezoelectric energy harvesting systems. Smart materials and structures, 15(6), 1499.

Zhao, H., Yu, J., \& Ling, J. (2010). Finite element analysis of cymbal piezoelectric transducers for harvesting energy from asphalt pavement. Journal of the Ceramic Society of Japan, 118(1382), 909-915.

\section{Copyrights}

Copyright for this article is retained by the author(s), with first publication rights granted to the journal.

This is an open-access article distributed under the terms and conditions of the Creative Commons Attribution license (http://creativecommons.org/licenses/by/4.0/). 